\section{COVID-19 y su trascendencia en la atención dental: revisión y actualización de la literatura}

\section{COVID-19 and its significance in dental care: review and update of the literature}

\section{Artículo de Revisión}

Jorge Luis Mija Gómez 1, a

${ }^{1}$ Universidad Nacional Mayor de San Marcos. Lima, Perú.

a Maestro en Estomatología.

\title{
Correspondencia:
}

Jorge Luis Mija Gómez: jlmijagomez@gmail.com Jr. Pasco 3353 2do piso. San Martin de Porres, Lima, Perú.

ORCID: 0000-0001-7728-9402

\section{Editor:}

Juan Carlos Cuevas-González

Universidad Autónoma de Ciudad Juárez, México.

Conflicto de intereses: el autor declara no tener conflictos de interés.

Fuente de financiamiento: autofinanciado.

Recibido: 02/06/20

Aceptado: 20/06/20

Publicado: 04/08/20

\begin{abstract}
Resumen
A fines del 2019 se presentaron 27 casos de neumonía atípica de etiología desconocida en la ciudad de Wuhan, China. Los síntomas de los pacientes que padecían la nueva neumonía viral fueron fiebre, tos seca, disnea y fatiga. El virus causante fue nombrado coronavirus de tipo 2 causante del síndrome respiratorio agudo severo (SARS-CoV-2) y la enfermedad fue nombrada en febrero de 2020 por la Organización Mundial de la Salud como enfermedad por coronavirus COVID-19. Las rutas de contagio de persona a persona incluyen transmisión directa por inhalación de gotitas de tos, estornudos, y transmisión indirecta, por medio de fómites que hagan contacto con las membranas mucosas orales, nasales u oculares. Los odontólogos debido a la naturaleza única de los procedimientos que realizan, tienen un alto riesgo de infección cruzada del COVID-19, ya que al trabajar en la boca del paciente está expuestos a una gran cantidad de gotas de saliva y aerosoles producidos durante la atención dental. La pandemia del COVID-19 requiere que el odontólogo tenga una preparación especial para la prevención del contagio en la consulta dental. El odontólogo también debe actualizarse sobre cómo el COVID-19 se relaciona con la profesión para estar bien preparado y ser miembro activo en las medidas sanitarias para controlar la pandemia. El objetivo de esta revisión es presentar información actualizada sobre el COVID-19, y dar soporte científico para el uso de las diferentes estrategias para minimizar el riesgo de contaminación cruzada en la práctica dental durante la pandemia de COVID-19.
\end{abstract}

Palabras clave: COVID-19; SARS-CoV-2; Atención dental; Bioseguridad (fuente: DeCS BIREME).

\section{Abstract}

By the end of 2019, 27 atypical pneumonia cases of unknown etiology were presented in Wuhan, China. The symptoms of the patients suffering from the new viral pneumonia were fever, dry cough, dyspnea, and fatigue. The virus was named coronavirus type 2 causing severe acute respiratory syndrome (SARS-CoV-2) and the disease was named in February 2020 by the World Health Organization as coronavirus disease COVID-19. 
The routes of transmission from person to person include direct transmission by inhalation of cough droplets, sneezing, and indirect transmission, through fomites that contact the oral, nasal, or ocular mucous membranes. Dentists, due to the unique nature of the procedures they perform, have a high risk of COVID-19 cross-infection, since when working in the patient's mouth they are exposed to a large number of saliva drops and aerosols that are produced during dental treatments. The COVID-19 pandemic requires dentists with special preparation for the prevention of contagion in the dental office. The dentist should also be updated on how COVID-19 relates to the profession to be well prepared and to be an active member in sanitary measures to control the pandemic. This review aims to present updated information on COVID-19 and to provide scientific support for the use of different strategies to minimize the risk of cross-contamination in dental practice during the COVID-19 pandemic.

Keywords: COVID-19; SARS-CoV-2; Dental care; Biosecurity (source: MeSH NLM).

\section{Introducción}

A fines de diciembre de 2019 se identificaron $27 \mathrm{ca}$ sos de neumonía atípica de etiología desconocida en la ciudad de Wuhan, provincia de Hubei en China ${ }^{1}$. Los síntomas clínicos típicos de los pacientes que padecían la nueva neumonía viral fueron fiebre, tos y mialgia o fatiga con tomografía computarizada (TC) torácica anormal, y los síntomas menos comunes fueron producción de esputo, dolor de cabeza, hemoptisis y diarrea. Investigadores chinos mediante exámenes virales, obtenidos de hisopado de garganta, aislaron rápidamente un nuevo virus y secuenciaron su genoma (29 903 nucleótidos), lo que descartó la presencia de virus comunes que causan neumonía, como los virus de la influenza, los adenovirus y los coronavirus asociados con el síndrome respiratorio del Medio Oriente (MERS) y el síndrome respiratorio agudo severo (SARS). Este nuevo patógeno fue nombrado inicialmente como "nuevo coronavirus 2019" (2019-nCoV) ${ }^{2}$ y oficialmente nombrado por el Comité Internacional de Taxonomía de Virus (ICTV) como coronavirus de tipo 2 causante del síndrome respiratorio agudo severo (SARS-CoV-2) debido al análisis filogenético y taxonómico de este novedoso coronavirus, y la enfermedad fue nombrada el 11 de febrero de 2020, por la Organización Mundial de la Salud (OMS), como enfermedad por coronavirus COVID-19 ${ }^{3}$.

Debido a la rápida diseminación por diferentes países, la epidemia de COVID-19 fue declarada el 30 de enero de 2020 por la OMS una emergencia de salud pública de preocupación internacional ${ }^{4}$, y el 11 de marzo preocupados por el nivel de propagación del COVID-19 la OMS lo declara como una pandemia de amenaza para la salud pública ${ }^{5}$.

Al 01 de junio de 2020, la OMS ha notificado 6057 853 casos confirmados de COVID-19 a nivel mundial, incluidas 371166 muertes ${ }^{6}$. En el Perú, el primer caso de COVID-19 se confirmó el 06 de marzo de 2020, y para el 27 de mayo, según datos oficiales, se habían reportado ya 135905 resultados positivos los cuales causaron la muerte de 3983 personas ${ }^{7}$.

Los odontólogos debido a la naturaleza única de los procedimientos que realizan, tienen un alto riesgo de infección cruzada del COVID-19, ya que al trabajar en la boca del paciente están expuestos a una gran cantidad de gotas de saliva (Flügge) ${ }^{8}$ y producción de aerosoles con gran potencial de transmitir el virus ${ }^{9}$. $\mathrm{Pa}-$ cientes asintomáticos pueden transmitir el COVID-19 ${ }^{10}$ lo que presenta un desafío aún mayor en el control y la prevención de la enfermedad. Por tanto, mientras dure la pandemia por COVID-19, solo se debe realizar tratamientos dentales de urgencia, para prevenir la transmisión del COVID-19 entre odontólogo, pacientes y personal del consultorio. Esta revisión tiene el objetivo de presentar aspectos importantes del COVID-19 y su relación con la práctica odontológica, haciendo énfasis en las medidas preventivas para evitar la infección cruzada durante los tratamientos de urgencia en el consultorio dental.

\section{COVID-19}

La enfermedad conocida como COVID-19 es causada por el virus SARS-CoV-2, este es un virus zoonótico, que, al identificar y comparar su secuencia de nucleótidos se encontró una homología del 96,2\% entre su ARN y la del coronavirus Bat-CoV-RaTG13 presente en el murciélago de herradura chino (Rhinolophus sinicus), por lo que se considera a este murciélago como el huésped natural del virus y al pangolín como el probable huésped intermedio en la ruta de transmisión de animal a humano, seguido de contagio masivo de humano a humano ${ }^{11,12}$. El SARS-CoV-2 tiene una homología del $80 \%$ con el SARS-CoV, de ahí que fue nombrado inicialmente como nuevo coronavirus $2019-\mathrm{nCoV}^{3}$.

Los coronavirus pertenecen a la familia Coronaviridae, tienen un material genético de ARN de cadena sencilla (monocatenario) de polaridad positiva [(+) ssARN], con un tamaño de 27-32 kilobases, y cuatro proteínas estructurales, de las cuales la proteína Spike (S) representan las estructuras más grandes del virus y son esenciales para la entrada en las células huésped ${ }^{3,13}$ (Figura 1). Los coronavirus se pueden diferenciar en cuatro géneros: alfa, beta, delta y gamma, de los cuales los coronavirus de tipo alfa y beta infectan a los humanos, principalmente los sistema respiratorio, gastrointestinal y sistema nervioso central. Estudios filogenéticos demuestran que los betacoronavirus incluye al SARS-CoV, el MERS-CoV y el agente causal COVID-19 ${ }^{14}$. 


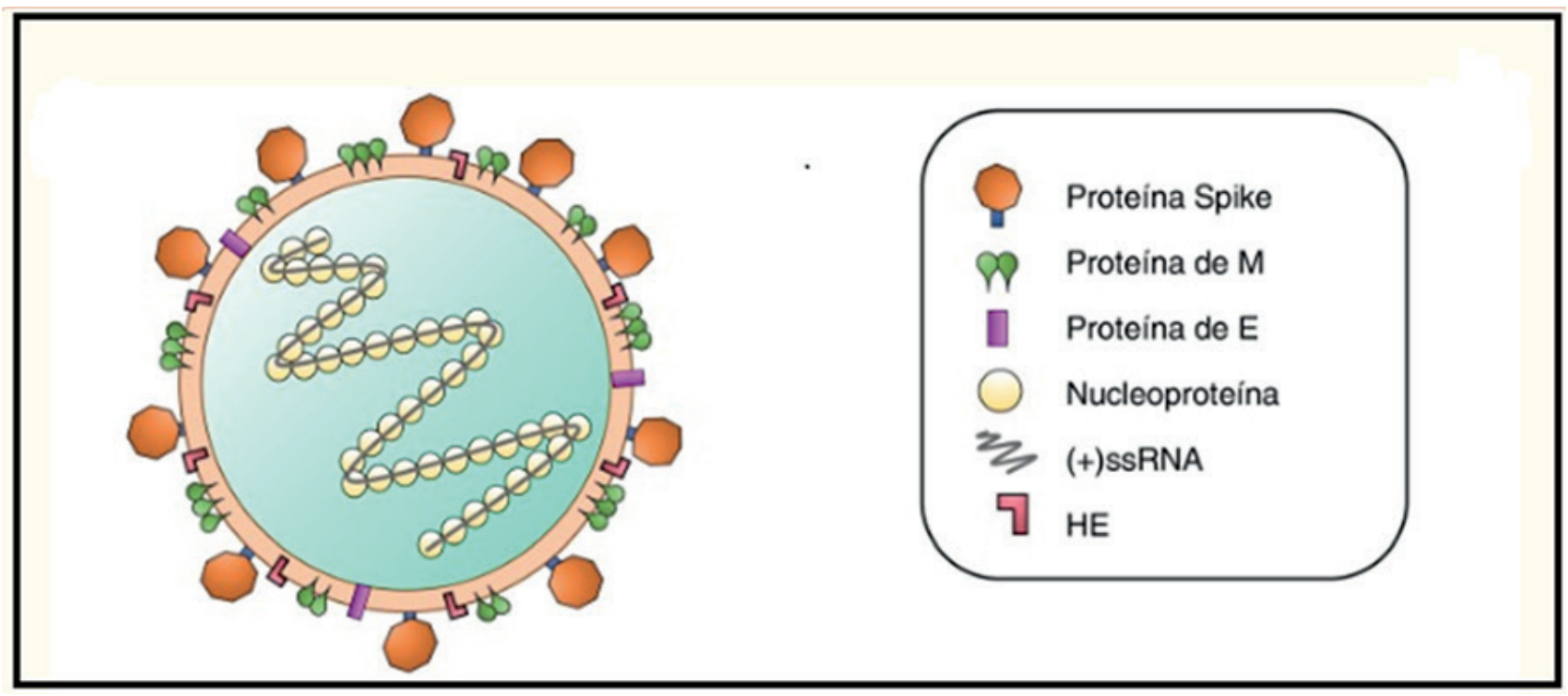

Figura 1. Partícula de coronavirus, (material genético de ARN de cadena sencilla de polaridad positiva), constituido por una nucleocápside y esta a su vez compuesta por (+) ssARN y la Nucleoproteína. ( tomado de: Palacios M, Santos E, Velázquez M, León M. COVID-19, una emergencia de salud pública mundial. [published online ahead of print, 2020 Mar 20]. Rev Clin Esp. 2020; S0014-2565(20)30092-8.)

\section{Rutas de contagio}

El COVID-19 se transmite directamente de persona a persona a través de las gotitas de Flügge (de 0,5 a 10 $\bigotimes \mathrm{m})$ originadas al hablar, toser o estornudar por la persona infectada ${ }^{11,15,16}$. Estas gotitas pueden permanecer suspendidas en el aire hasta por 30 minutos y a una distancia máxima de 1 metro, luego se depositan rápidamente, por tanto, para ser vehículo de transmisión debe haber un contacto cercano con la persona infectada ${ }^{8,17}$. También es probable el contagio indirecto por contacto con fómites en el entorno de un infectado ${ }^{15}$, y luego de este contacto se lleve material infectado a la mucosa oral, nasal y también esta reportado el contagio a través de la conjuntiva del ojo ${ }^{18}$. Respecto a la transmisión intrauterina del SARS-CoV-2, la evidencia actual sugiere que la transmisión vertical es poco probable ${ }^{19}$.

La enzima convertidora de angiotensina 2 (ACE2) es el principal receptor de las células huésped del SARS$\mathrm{CoV}-2$ y desempeña un papel crucial en la entrada del virus en la célula para causar la infección final ${ }^{20}$. La proteína $S$ del SARS-CoV-2 es la responsable de unirse al receptor $\mathrm{ACE} 2$, este receptor se encuentra altamente expresado en las células epiteliales de todo el tracto respiratorio, lengua y de las glándulas salivales, lo que explica el riesgo potencialmente alto de infección a través de la cavidad oral ${ }^{3,15}$.

\section{Periodo de incubación}

El período de incubación del COVID-19 es de 5 a 6 días en promedio, llegando en algunos casos a los 14 días ${ }^{21,22}$, que son los días recomendados para la observación médica y cuarentena de potenciales infectados. Este periodo de incubación relativamente largo, con ausencia de cualquier sintomatología, representa un desafío para el personal de salud en la lucha por controlar la propagación del COVID-19.

\section{Características clínicas}

Los síntomas iniciales son presencia de fiebre, tos seca, congestión nasal, fatiga y otros signos de infección del tracto respiratorio alto. La mayoría de los pacientes con COVID-19 presentan sintomatología relativamente leve, pero en aproximadamente la cuarta parte de los casos, la infección puede progresar a enfermedad grave con disnea y congestión pulmonar severa. La insuficiencia multiorgánica puede dar lugar a insuficiencia respiratoria, shock, síndrome de dificultad respiratoria aguda, arritmia, lesión aguda del miocardio, lesión hepática aguda y sepsis, incluso la muerte ${ }^{11}$. Esta progresión está asociada a un aumento extremo de citosinas pro-inflamatorias, incluidas interleuquinas (IL-1B, IL-2, IL-6, IL-7, IL-10, IL-12), interferones (IFN $\alpha$, IFN $\gamma$ ), factor de necrosis tumoral alfa (TNF- $\alpha$ ), factor de crecimiento transformante beta (TGF- $\beta$ ) y quimioquinas (CCL2, CCL3, CCL5, CXCL8, CXCL10). Este aumento llamado tormenta de citoquinas, conlleva a la muerte por una respuesta inflamatoria sistémica no controlada ${ }^{23-25}$. En pacientes de edad avanzada y pacientes con existencia de comorbilidades, principalmente hipertensión, diabetes y cardiopatía isquémica, el riesgo de complicaciones graves aumenta ${ }^{26,27}$.

Está reportado que aproximadamente el $80 \%$ de los pacientes con COVID-19 son asintomáticos o con sintomatología muy leve, sobretodo en pacientes niños y jóvenes. Los portadores asintomáticos pueden provocar una transmisión de persona a persona y deben considerarse una fuente de infección del COVID-19 ${ }^{28-30}$. 
Las manifestaciones cutáneas son menos frecuentes, pero en casos agudos de COVID-19 se puede presentar erupción eritematosa, urticaria localizada o generalizada, sin embargo, puede ser difícil distinguir la causa subyacente (infección viral o la medicación prescrita) ${ }^{31}$. En niños con COVID-19 se ha reportado casos raros de eritema multiforme, síndrome hiperinflamatorio multisistémico similar a la enfermedad de Kawasaki ${ }^{32,33}$.

\section{Manifestaciones bucales de COVID-19}

El genoma del SARS-CoV-2 se ha detectado en la saliva de la mayoría de los pacientes con COVID-19, lo que indica la posible infección de las glándulas salivales. En algunos pacientes el SARS-CoV-2 se detectó solo en la saliva, pero no en el aspirado nasofaríngeo. La saliva también se ha utilizado para detectar al virus en pacientes hospitalizados sin fiebre o síntomas respiratorios ${ }^{34,35}$.

La pérdida del gusto y el olfato se ha reportado como uno de los síntomas de COVID - 19. La mayoría de los pacientes con estos síntomas (91\%) informaron la aparición de alteraciones del gusto aun antes de los síntomas respiratorios. El trastorno del gusto podría explicarse por la alta expresión de ACE2 en el dorso de la lengua, rica en papilas gustativas ${ }^{27}$.

Se ha reportado presencia de gingivitis descamativa, úlceras y ampollas compatibles con estomatitis herpética recurrente en la mucosa palatina, aparentemente asociado a COVID-19, así como sensación de quemazón y dolor de la orofaringe ${ }^{36}$ (Figura 2).

Pacientes con periodontitis moderada o grave no tratada puede empeorar en casos severos de COVID-19, en los que un estado inflamatorio persistente parece actuar como un desencadenante de la cascada de coagulación y se asocia con mayores niveles de productos de degradación de fibrinógeno (por ejemplo, dímero D) ${ }^{37}$. También se reportó un aumento espontáneo en la prevalencia de enfermedad periodontal necrotizante
(EPN), en relación al aumento de casos confirmados de COVID-19. La etiología de la EPN puede estar asociada con coinfecciones bacterianas que ocurren intraoralmente en pacientes con COVID-19. Los análisis metagenómicos de los infectados con frecuencia detectan lecturas bacterianas anormalmente altas de Prevotella intermedia, además de géneros patógenos comunes implicados en la aparición y progresión de enfermedades orales como: estreptococos, Fusobacterium, Treponema y Veillonella. P. intermedia se considera una especie bacteriana etiológica importante para varias lesiones periodontales agudas, que junto con las especies de Fusobacterium y Treponema, constituyen una gran proporción de la microbiota presente en las lesiones EPN ${ }^{38}$.

\section{Atención odontológica en tiempos de COVID-19}

Los odontólogos son los profesionales de mayor riesgo de transmisión de COVID-19, ya que por la naturaleza de la atención dental, desde el diagnóstico al tratamiento el odontólogo y el paciente se encuentran cara a cara, además que la producción de aerosoles durante procedimientos dentales debido al uso de piezas de mano de alta y baja velocidad, raspadores ultrasónicos, jeringa triple, coloca al odontólogo, personal que trabaja en el consultorio y pacientes en un alto riesgo de infección cruzada (Figura 3). La transmisión de COVID-19 en la consulta dental se puede dar por cuatro rutas principales: (1) exposición directa a secreciones respiratorias del paciente; (2) contacto indirecto con superficies o instrumentos contaminados; (3) inhalación de suspensión de virus en el aire; y (4) contacto de la mucosa (nasal, oral y conjuntival) con gotitas y aerosoles que contienen infección que se impulsan al toser y hablar sin mascarilla ${ }^{15}$.

Los pacientes con COVID-19 no deben ser tratados en un entorno de atención dental regular, sino en un ambiente hospitalario en una sala de aislamiento de presión negativa. Los pacientes asintomáticos (portadores) pueden
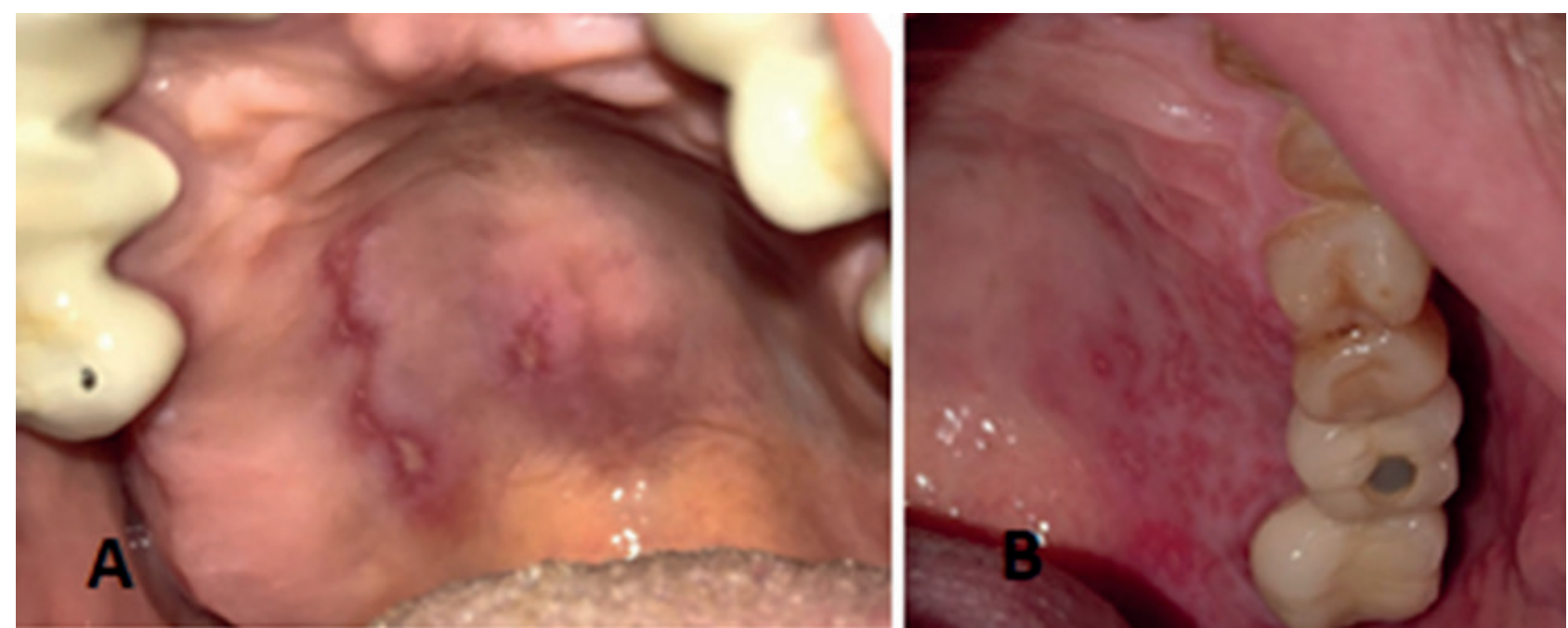

Figura 2. A. Múltiples úlceras de color naranja con halo eritematoso y distribución simétrica en el paladar duro derecho del paciente. B. Múltiples úlceras amarillentas con un halo eritematoso en el paladar duro izquierdo del paciente.

(tomado de: Carreras-Presas M, et al.) 


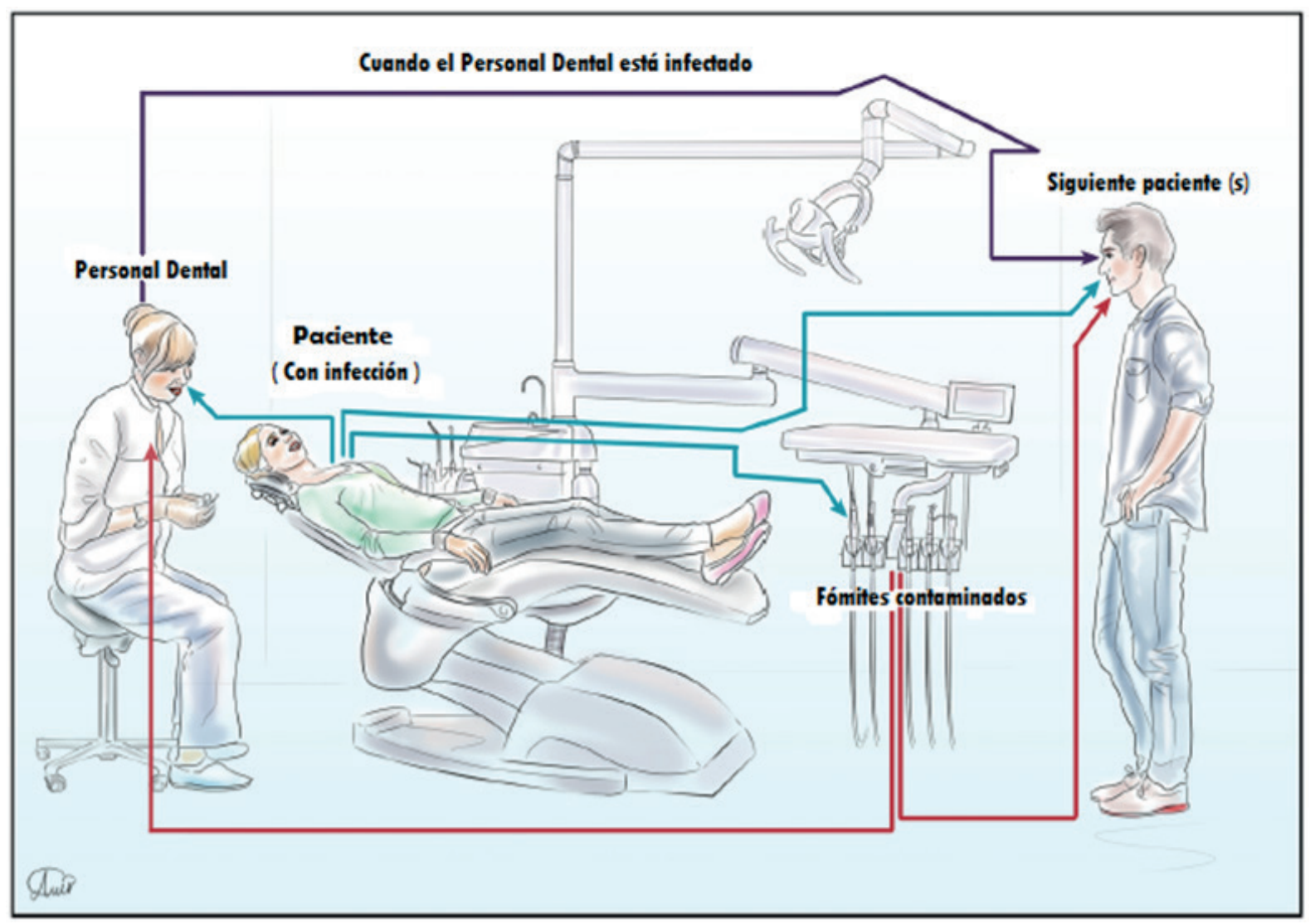

Figura 3. Dinámica de la infección cruzada en el consultorio dental. (Adaptado de Fallahi H, et al. 2020)

presentarse para recibir tratamiento dental y por la dificultad de identificarlos son de gran riesgo para la transmisión de COVID-19. Esta característica epidemiológica de COVID-19 ha hecho su control extremadamente difícil, por lo que los tratamientos electivos deben posponerse y solo realizar tratamientos de urgencia en el consultorio dental, siguiendo protocolos de control de infección estrictos. La decisión sobre la realización del tratamiento debe tomarse con el consentimiento apropiado del paciente, quien firmara el consentimiento informado en el que se debe explicar los riesgos de la atención dental en tiempos de la pandemia COVID-19 ${ }^{39}$.

El odontólogo y todo el personal que trabaje en la consulta dental deben realizarse la prueba diagnóstica de COVID-19 para descartar algún positivo que pueda contagiar a los pacientes, sin embargo, un resultado negativo no quita la posibilidad de infección posterior a la toma de la muestra, por tanto un resultado negativo no debe descuidar la implementación de las medidas de prevención y control del COVID-19.

\section{Evaluación del paciente (triaje)}

Con el propósito de controlar la infección por COVID-19, la medida preventiva fundamental radica en el triaje de pacientes que requieran atención dental de urgencia en el consultorio. El examen inicial por teléfono para identificar pacientes con sospecha o posible infección con COVID-19 se puede realizar de forma remota al momento de programar las citas (Figura 4). Las tres preguntas más relevantes para el triaje inicial deben incluir cualquier exposición a una persona con diagnóstico conocido o sospecha de COVID-19, cualquier historial de viaje reciente a un área con alta incidencia de COVID-19 o presencia de cualquier síntoma de enfermedad respiratoria y/o fiebre. Una respuesta positiva a cualquiera de las tres preguntas debe generar preocupación inicial, y el cuidado dental electivo debe diferirse por al menos 2 semanas. Se debe alentar a estos pacientes a que realicen la cuarentena y se comuniquen con su médico de atención primaria por teléfono ${ }^{40}$.

Debido a que solo se debe de realizar tratamientos de urgencia, una videollamada o un mensaje con foto es una herramienta muy útil para determinar la necesidad de tratamiento del paciente, y así evitar el traslado del paciente al consultorio encaso no sea una verdadera urgencia. Aunque su impacto en el entorno clínico ha sido poco investigado, la aplicación WhatsApp Messenger (Facebook Inc., Mountain View, California) se encuentra entre las herramientas de comunicación más utilizadas por personas de toda edad ${ }^{41}$. El primer paso siempre debe ser la asistencia virtual, y WhatsApp puede considerarse una buena herramienta para hacerlo. La asistencia virtual se puede realizar mediante el uso de fotos y videos. Se recomienda hacer un triaje a través de WhatsApp para analizar las urgencias reales que se puedan presentar y evaluar la necesidad de una cita presencial o la posible atención de forma remota ${ }^{42,43}$. 


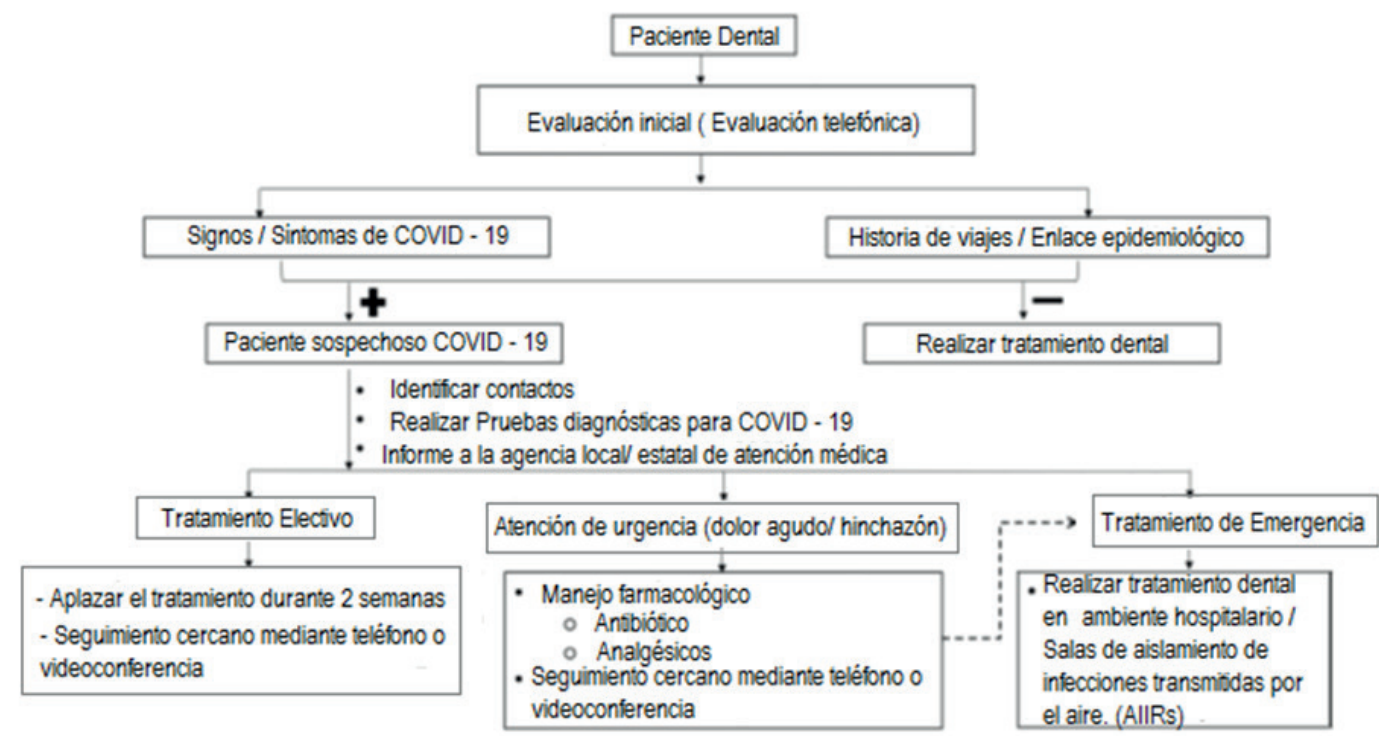

Figura 4. Flujograma de atención a pacientes durante la pandemia COVID-19. (Adaptado de: Ather A et al.)

Al llegar el paciente a la consulta dental, se debe hacer la desinfección del calzado y colocarse botas descartables. Se debe medir la temperatura corporal del paciente utilizando un termómetro infrarrojo a distancia, los pacientes que presentan fiebre $\left(38^{\circ} \mathrm{C}\right)$ y / o síntomas de enfermedad respiratoria deben recibir indicaciones para que cumplan cuarentena de 2 semanas y se debe posponer todo tratamiento electivo, y en caso de tener una urgencia que no se pueda controlar con medidas farmacológicas, se debe derivar al paciente a un ambiente hospitalario donde pueda atenderse en una sala con presión negativa. En caso el paciente no presente ninguna sintomatología que nos indique posible contagio de COVID-19, antes de recibir atención dental, debe completar un formulario de historial médico detallado, un cuestionario de detección COVID-19 y la firma del consentimiento informado detallando los posibles riesgos de atención dental y COVID-19 ${ }^{40,44}$. Es de suma importancia que los pacientes se programen con cita previa, con un espacio de tiempo de seguridad entre consultas dentales, evitando el contacto o incluso la proximidad con otros pacientes.

\section{Uso de equipo de protección personal (EPP)}

El EPP consiste en prendas para proteger a todo el personal, incluido limpieza y seguridad que este potencialmente expuesto a contagio. El EPP estándar consiste en guantes, mascarilla y mandilón. Sin embargo, en caso de infecciones transmitidas por el aire como el COVID-19, se debe usar equipo adicional que incluya guantes, gorro, mascarillas o respiradores, gafas, máscaras protectoras, trajes de aislamiento y ropa protectora, diseñados para proteger la piel y las membranas mucosas de los ojos, la boca y nariz ${ }^{27}$. Si bien es cierto que el uso de EPP en la consulta es lo que más resalta para el paciente, no es más que una de las medidas para prevenir infecciones cruzadas y no se debe considerar como la medida principal de prevención; si no está acompañado de medidas administrativas y técnicas eficaces, como por ejemplo el triaje a distancia, lavado de manos, la utilidad de los EPP es limitada ${ }^{45}$.

El uso de guantes no sustituye el lavado de manos y debido a la baja resistencia del SARS-CoV-2 a los detergentes, es la medida más importante en el control de la infección. El lavado de manos debe cumplir con la regla del "dos antes y tres después" que incluye antes de tocar a un paciente, antes de realizar cualquier procedimiento aséptico, después de exposición a fluidos corporales del paciente, después de tocar a un paciente y después de tocar los alrededores del paciente o elementos que puedan estar contaminados ${ }^{46}$.

El uso de mascarillas como protección respiratoria ha demostrado efectividad en el control de infección del COVID-19. Un concepto importante sobre la protección respiratoria a bioaerosoles es que los microorganismos son partículas que poseen forma, tamaño y peso, por lo que pueden ser filtrados. Las gotas son consideradas mayores a $5 \mu \mathrm{m}$ y los aerosoles menores a $5 \mu \mathrm{m}$. Dentro de la protección respiratoria para el manejo de microorganismos tenemos las mascarillas quirúrgicas o cubrebocas y los respiradores. Aunque las mascarillas quirúrgicas tienen cierta capacidad de filtración, no deben usarse cuando se atienden pacientes con enfermedades causadas por aerosoles (menor a $5 \mu \mathrm{m}$ ). Estas mascarillas no están diseñadas para brindar un sello facial; por tanto, no filtran alrededor del borde de la mascarilla cuando el usuario inhala, por lo que la transmisión ocurre por la diseminación de material infeccioso de tamaño tal que es respirable. Los respiradores son equipos que filtran todo el aire que respira el usuario, aunque con mayor o menor eficiencia según el modelo y especificaciones. En Estados Unidos los respiradores certificados por el Instituto Nacional para la Seguridad y Salud Ocupacional (NIOSH) son los N95 y tienen un mínimo de filtración del $95 \%$ para las partículas más penetrantes $(0,1$ a 0,3 
$\mu \mathrm{m})$. El respirador N95 tiene que ajustar bien sobre la cara para prevenir la filtración de aire contaminado. Estos respiradores vienen en diferentes tamaños, se debe usar el que se ajuste mejor al rostro ${ }^{47-49}$. Es importante destacar que los aerosoles pueden permanecer suspendidos en el aire hasta por 30 minutos, por lo tanto, quitar la máscara antes de 30 minutos en este entorno puede aumentar el riesgo de contacto con partículas contaminadas ${ }^{50}$. Se debe evitar que el respirador haga contacto con el labio al momento de retirarlo.

La pandemia de COVID-19 ha provocado una gran escasez de respiradores N95, lo que ha llevado a realizar estudios que demuestren la efectividad de su desinfección para reusarlos, se encontró que el calor seco inactiva el virus del COVID-19 luego de 5 minutos a $70^{\circ} \mathrm{C}$ y es uno de los métodos más seguros y que no produce deterioro del respirador, pudiendo realizarse hasta por 5 veces, siempre que el respirador no haya sido contaminado con fluidos del paciente. La irradiación ultravioleta (UV) fue una opción secundaria, sin embargo, la luz UV puede afectar la resistencia del material y el posterior sellado de los respiradores. Finalmente, los tratamientos que involucran líquidos y vapores requieren precaución, ya que el vapor, el alcohol y el hipoclorito pueden conducir a la degradación de la eficiencia de filtración, dejando al usuario vulnerable a los aerosoles virales ${ }^{51,52}$.

\section{Uso de colutorios}

Debido a que el SARS-CoV-2 es bastante sensible a la oxidación, se debe proporcionar un enjuague bucal con agentes oxidantes al paciente antes de comenzar el procedimiento dental, para disminuir la carga viral en la saliva de un paciente infectado. Se recomienda peróxido de hidrógeno al $1 \%$, para obtener $15 \mathrm{~mL}$ de este colutorio, se mezcla $5 \mathrm{~mL}$ de peróxido de hidrógeno a $10 \mathrm{Vol}$. adicionando $10 \mathrm{~mL}$ de agua destilada. También puede usarse yodopovidona al $0.2 \%$. La clorhexidina parece no tener efecto sobre el SARS-CoV-2, por lo que no es aconsejable su uso ${ }^{53,54}$.

\section{Reducción de producción de aerosoles.}

El riesgo más grande de transmisión de COVID-19 durante la atención dental, es a través de la generación de aerosoles, ya que el virus puede permanecer viable e infectar ya sea por inhalación o por contacto con la mucosa oral, nasal o la conjuntiva del ojo. El uso de instrumental rotatorio y jeringa triple debe evitarse en lo posible, ya que crea un spray visible o aerosol que contiene principalmente gotas de agua, saliva, sangre, microorganismos, y otros desechos, que se van a precipitar por la gravedad contaminando las superficies expuestas del consultorio ${ }^{55,56}$. En caso no se pueda evitar el uso de la pieza de mano, esta debe tener una válvula antirretracción que evite la aspiración y expulsión de desechos y fluidos durante los procedimientos dentales, ya que se ha determinado que microorganismos pueden contaminar los tubos de agua y aire de la unidad dental si es que no se cuenta con válvula antirretracción ${ }^{57}$.
Se debe evitar la toma de radiografías intraorales, para prevenir tos o reflejo nauseoso en el paciente, lo que generaría aerosoles. Las radiografías extraorales y la TC son alternativas a tomar en cuenta ${ }^{50}$.

\section{Aislamiento absoluto con dique de goma}

El aislamiento absoluto con dique de goma, debido a la creación de una barrera en la cavidad oral reduce efectivamente la generación de gotas y aerosoles mezclados con saliva y/o sangre del paciente en un $70 \%$. Después de la colocación del dique, se requiere adicionalmente de una succión de alto volumen para evitar al máximo la propagación de aerosoles y salpicaduras. El aislamiento absoluto debe ser de rutina en todos los procedimientos dentales que lo permitan ${ }^{15,42}$.

\section{Desinfección de superficies del consultorio}

La investigación ha demostrado que los coronavirus pueden permanecer en superficies de metal, vidrio y plástico de manera activa a temperatura ambiente de 2 horas hasta 9 días. Por lo tanto, como las superficies en las clínicas dentales sirven como lugares de depósito para gotas y aerosoles mezclados con la saliva y / o sangre de los pacientes, pueden ayudar efectivamente a propagar la infección. Pruebas recientes indican que el SARS-CoV-2 puede ser vulnerable a biocidas como el hipoclorito de sodio al $0,1 \%$, el peróxido de hidrógeno al $0,5 \%$, al etanol de 60 a $75 \%$, glutaraldehído al 2,5\%, formaldehído $1 \%$ y compuestos de amonio fenólico y cuaternario si se utilizan de acuerdo con las instrucciones del fabricante. El digluconato de clorhexidina al $0,02 \%$ es ineficaz ${ }^{15,59}$.

El uso de luz ultravioleta (UV) para la desinfección del consultorio no ha sido probada para inactivar al SARS$\mathrm{CoV}-2$, pero si hay evidencia de la inactivación por luz UV (especialmente la UV-C) de los coronavirus MERS y SARS, genéticamente muy parecidos al SARS-CoV-2. En caso se cuente con luz UV-C para la desinfección de las superficies del consultorio, se debe usar como un complemento y no debe remplazar de ninguna manera la desinfección con agentes biocidas ${ }^{60-62}$.

\section{Conclusiones}

La declaración de pandemia de COVID-19 por la OMS nos pone en un escenario pocas veces imaginado, y el odontólogo debe conocer las características del SARS-CoV-2, las rutas de transmisión, manifestaciones clínicas iniciales que lleven a identificar a los pacientes infectados y lo más importante, las medidas a tomar para interrumpir la cadena de transmisión. El odontólogo desde su consulta privada debe participar activamente en las políticas de salud pública, identificando posibles contagiados, orientándolos en las medidas para no propagar la infección y derivándolos para la atención especializada. La atención dental debe restringirse exclusivamente a tratamientos de emergencia y urgencia, siguiendo estrictamente los protocolos de bioseguridad y medidas específicas para el control de COVID-19. 


\section{Referencias bibliográficas}

1. Sohrabi C, Alsafi Z, O'Neill N, Khan M, Kerwan A, Al-Jabir A, et al. World Health Organization declares global emergency: A review of the 2019 novel coronavirus (COVID-19). Int J Surg. 2020; 76:71-76. DOI: 10.1016/j.ijsu.2020.02.034.

2. Jung S, Kinoshita R, Thompson R, Linton N, Yang Y, Akhmetzhanov A, et al. Epidemiological Identification of A Novel Pathogen in Real Time: Analysis of the Atypical Pneumonia Outbreak in Wuhan, China, 2019-2020. J Clin Med. 2020;9(3):637. DOI:10.3390/jcm9030637

3. Peng X, Xu X, Li Y, Cheng L, Zhou X, Ren B. Transmission routes of 2019-nCoV and controls in dental practice. Int J Oral Sci. 2020;12:9. DOI: 10.1038/s41368020-0075-9.

4. World Health Organization (WHO) Declaración del Director General de la OMS sobre la reunión del Comité de Emergencia del Reglamento Sanitario Internacional acerca del nuevo coronavirus (2019-nCoV). [Consultado el 10 de abril de 2020]. Accesible en: https://www.who. int/es/dg/speeches/detail/who-director-general-s-statement-on-ihr-emergency-committee-on-novel-coronavirus-(2019-ncov)

5. World Health Organization (WHO) Director-General's opening remarks at the media briefing on COVID-19 - 11 March 2020. [Consultado el 15 de abril de 2020]. Accesible en: https://www.who.int/dg/speeches/detail/ who-director-general-s-opening-remarks-at-the-mediabriefing-on-covid-19---11-march-2020.

6. World Health Organization (WHO). Coronavirus disease (COVID-19) Situation Report - 133. [Consultado el 02 de junio de 2020]. Accesible en: https://www.who.int/docs/default-source/coronaviruse/situation-reports/20200601-covid-19-sitrep 133.pdf?sfvrsn=9a56f2ac_4.

7. Ministerio de Salud. (MINSA). Comunicado N ${ }^{\circ} 113$. Casos confirmados por Coronavirus COVID-19 ascienden a 135905 en el Perú. [Consultado el 01 de junio de 2020]. Accesible en: https://www.gob.pe/institucion/minsa/noticias/165852-minsa-casos-confirmados-por-coronavirus-covid-19-ascienden-a-135-905-en-el-peru-comunicado-n-113.

8. Murillo G. Las gotitas de Flügge. Rev Med Inst Mex Seguro Soc. 2009;47(3):290.

9. Watanabe A, Tamaki N, Yokota K, Matsuyama M, Kokeguchi S. Use of ATP bioluminescence to survey the spread of aerosol and splatter during dental treatments. J Hosp Infect. 2018;99(3):303-305. DOI: 10.1016/j. jhin.2018.03.002

10. Jiang X, Zhang X, Zhao X, Li C, Lei J, Kou Z. Transmission Potential of Asymptomatic and Paucisymptomatic SARS-CoV-2 Infections: A Three-Family Cluster Study in China. J Infect Dis. 2020;221(12):1948-52. DOI: 10.1093/infdis/jiaa206.

11. Meng L, Hua F, Bian Z. Coronavirus Disease 2019 (COVID-19): Emerging and Future Challenges for Dental and Oral Medicine. J Dent Res. 2020;99(5):481-487. DOI: $10.1177 / 0022034520914246$.

12. Reina J. El SARS-CoV-2, una nueva zoonosis pandémica que amenaza al mundo. Vacun. 2020;21(1):17-22. DOI: $10.1016 /$ j.vacun.2020.03.001
13. Palacios M, Santos E, Velázquez M, León M. COVID-19, una emergencia de salud pública mundial. [published online ahead of print, 2020 Mar 20]. Rev Clin Esp. 2020;S0014-2565(20)30092-8. DOI: 10.1016/j. rce.2020.03.001

14. Baghizadeh M. What dentists need to know about COVID-19. Ora.l Oncol. 2020;105:104741. DOI: 10.1016/j.oraloncology.2020.104741.

15. Fallahi H, Keyhan S, Zandian D, Kim S, Cheshmi B. Being a front-line dentist during the Covid-19 pandemic: a literature review. Maxillofac Plast Reconstr Surg. 2020;42(1):12. DOI: 10.1186/s40902-020-00256-5.

16. Sigua E, Bernal J, Lanata A, Sánchez C, Rodríguez J, Haidar Z, et al. COVID-19 y la Odontología: una Revisión de las Recomendaciones y Perspectivas para Latinoamérica. Int. J. Odontostomat. 2020;14(3):299309.

17. Aranaz J, Gea M, Requena J. Mitos y miedos: las precauciones frente a la gripe aviar las justifica el mecanismo de transmisión. Gac Sanit. 2006;20(5):410-3. DOI: $10.1157 / 13093212$.

18. Lu C, Liu X, Jia Z. 2019-nCoV transmission through the ocular surface must not be ignored. Lancet. 2020; Feb 22;395(10224):e39. DOI: 10.1016/S01406736(20)30313-5.

19. Chen H, Guo J, Wang C, Luo F, Yu X, Zhang W, et al. Clinical characteristics and intrauterine vertical transmission potential of COVID-19 infection in nine pregnant women: a retrospective review of medical records. Lancet. 2020; Mar 7;395(10226):809-815. DOI: 10.1016/ S0140-6736(20)30360-3.

20. Xu H, Zhong L, Deng J, Peng J, Dan H, Zeng X, et al. High expression of ACE2 receptor of 2019-nCoV on the epithelial cells of oral mucosa. Int J Oral Sci. 2020;12(1):8. DOI: 10.1038/s41368-020-0074-x

21. Backer J, Klinkenberg D, Wallinga J. Incubation period of 2019 novel coronavirus (2019-nCoV) infections among travellers from Wuhan, China, 20-28 January 2020. Euro Surveill. 2020;25(5):2000062. DOI: 10.2807/1560-7917.ES.2020.25.5.2000062.

22. Khader $\mathrm{Y}, \mathrm{Al}$ Nsour M, Al-Batayneh O, Saadeh R, Bashier H, Alfaqih M, et al. Dentists' Awareness, Perception, and Attitude Regarding COVID-19 and Infection Control: Cross-Sectional Study Among Jordanian Dentists. JMIR Public Health Surveill. 2020;6(2):e18798. DOI: 10.2196/preprints. 18798 .

23. Channappanavar R, Perlman S. Pathogenic human coronavirus infections: causes and consequences of cytokine storm and immunopathology. Semin Immunopathol. 2017;39(5):529-539. DOI:10.1007/s00281017-0629-x.

24. Ramírez M, Medina P, Morocho A. Enfermedad por coronavirus 2019 (COVID-19) y su repercusión en la consulta odontológica: una revisión. Odontol Sanmarquina. 2020;23(2):139-146. DOI: 10.15381/os.v23i2.17758.

25. Huang C, Wang Y, Li X, Ren L, Zhao J, Hu Y, et al. Clinical features of patients infected with 2019 novel coronavirus in Wuhan, China. Lancet. 2020; 395:497-506. DOI:10.1016/S0140-6736(20)30183-5. 
26. Tarazona-Santabalbina F, Martínez-Velilla N, Vidán M, García-Navarro J. COVID-19, adulto mayor y edadismo: errores que nunca han de volver a ocurrir. Rev Esp Geriatr Gerontol. 2020; S0211 139X(20)30059-7. DOI: $10.1016 /$ j.regg.2020.04.001.

27. Odeh N, Babkair H, Abu-Hammad S, Borzangy S, Abu-Hammad A, Abu-Hammad O. COVID-19: Present and Future Challenges for Dental Practice. Int J Environ Res Public Health. 2020;17(9):E3151. DOI: 10.3390/ijerph17093151.

28. Jiang X, Zhang X, Zhao X, Li C, Lei J, Kou Z, et al. Transmission potential of asymptomatic and paucisymptomatic SARS-CoV-2 infections: a three-family cluster study in China. J Infect Dis. 2020;221(12):1948-52. DOI: 10.1093/infdis/jiaa206.

29. Hu Z, Song C, Xu C, Jin G, Chen Y, Xu X, et al. Clinical characteristics of 24 asymptomatic infections with COVID-19 screened among close contacts in Nanjing, China. Sci China Life Sci. 2020;63(5):706-711. DOI: 10.1007/s11427-020-1661-4.

30. Bai Y, Yao L, Wei T, Tian F, Jin D, Chen L, et al. Presumed Asymptomatic Carrier Transmission of COVID-19 [published online ahead of print, $2020 \mathrm{Feb}$ 21]. JAMA. 2020;323(14):1406-1407. DOI:10.1001/ jama.2020.2565.

31. Estébanez A, Pérez-Santiago L, Silva E, Guillen-Climent S, García-Vázquez A, Ramón M. Cutaneous manifestations in COVID-19: a new contribution [published online ahead of print, 2020 Apr 15]. J Eur Acad Dermatol Venereol. 2020;10.1111/jdv.16474. DOI: 10.1111/ jdv. 16474 .

32. Labé P, Ly A, Sin C, Nasser M, Chapelon-Fromont E, Ben $\mathrm{P}$, et al. Erythema multiforme and Kawasaki disease associated with COVID-19 infection in children [published online ahead of print, 2020 May 26]. J Eur Acad Dermatol Venereol. 2020;10.1111/jdv.16666. DOI: 10.1111/JDV.16666.

33. Licciardi F, Pruccoli G, Denina M, Parodi E, Taglietto M, Rosati S, et al. SARS-CoV-2-Induced Kawasaki-Like Hyperinflammatory Syndrome: A Novel COVID Phenotype in Children [published online ahead of print, 2020 May 21]. Pediatrics. 2020; e20201711. DOI: 10.1542/peds.2020-1711.

34. To K, Tsang O, Chik-Yan Yip C, Chan K, Wu T, Chan J, et al. Consistent detection of 2019 novel coronavirus in saliva [published online ahead of print, $2020 \mathrm{Feb}$ 12]. Clin Infect Dis. 2020; ciaa149. DOI: $10.1093 / \mathrm{cid} /$ ciaa149.

35. Sabino-Silva R, Jardim A, Siqueira W. Coronavirus COVID-19 impacts to dentistry and potential salivary diagnosis. Clin Oral Investig. 2020;24(4):1619-1621. DOI: 10.1007/s00784-020-03248-x

36. Carreras-Presas M, Sánchez A, López-Sánchez A, Salas J, Pérez S. Oral vesiculobullous lesions associated with SARS-CoV-2 infection. Oral Dis. 2020;10.1111/ odi.13382. DOI: 10.1111/ODI.13382.

37. Vieira A. Oral Manifestations in Coronavirus Disease 2019 (COVID-19) [published online ahead of print, 2020 Jun 7]. Oral Dis. 2020;10.1111/odi.13463. doi:10.1111/odi.13463.
38. Patel J, Woolley J. Necrotizing periodontal disease: oral manifestation of COVID-19 [published online ahead of print, 2020 Jun 7]. Oral Dis. 2020;10.1111/odi.13462. DOI: 10.1111 /odi.13462.

39. Dave M, Seoudi N, Coulthard P. Urgent dental care for patients during the COVID-19 pandemic. Lancet. 2020;395(10232):1257. DOI: 10.1016/S01406736(20)30806-0.

40. Ather A, Patel B, Ruparel N, Diogenes A, Hargreaves K. Coronavirus Disease 19 (COVID-19): Implications for Clinical Dental Care. J Endod. 2020;46(5):584-595. DOI:10.1016/j.joen.2020.03.008.

41. Caprioglio A, Pizzetti G, Zecca P, Fastuca R, Maino G, Nanda R. Management of orthodontic emergencies during 2019-NCOV. Prog Orthod. 2020; Apr 7;21(1):10. DOI: $10.1186 / s 40510-020-00310-y$.

42. Krynskia L, Goldfarba G, Maglioc I. La comunicación con los pacientes mediada por tecnología: WhatsApp, e-mail, portales. El desafío del pediatra en la era digital. Arch Argent Pediatr 2018;116(4):554-559. DOI: 10.5546/aap.2018.e554.

43. Mars M, Scott R. WhatsApp in Clinical Practice: A Literature Review. Stud Health Technol Inform. 2016;231:82-90. DOI: 10.3233/978-1-61499-712-2-82.

44. Guiñez M. Impacto del COVID-19 (SARS-CoV-2) a Nivel Mundial, Implicancias y Medidas Preventivas en la Práctica Dental y sus Consecuencias Psicológicas en los Pacientes. Int. J. Odontostomat. 2020;14(3):271-278.

45. Huh S. How to train the health personnel for protecting themselves from novel coronavirus (COVID-19) infection during their patient or suspected case care. J Educ Eval Health Prof. 2020;17:10. DOI: 10.3352/jeehp.2020.17.10.

46. Yan Y, Chen H, Chen L, Cheng B, Diao P, Dong L, et al. Consensus of Chinese experts on protection of skin and mucous membrane barrier for health-care workers fighting against coronavirus disease 2019 [published online ahead of print, 2020 Mar 13]. Dermatol Ther. 2020; e13310. DOI: 10.1111/dth.13310.

47. Wang X, Pan Z, Cheng Z. Association between 2019$\mathrm{nCoV}$ transmission and N95 respirator use. J Hosp Infect. 2020;105(1):104-105. DOI: 10.1016/j. jhin.2020.02.021.

48. Castañeda J, Hernández H. Mascarilla N95: una medida útil en la prevención de la tuberculosis pulmonar. Acta Pediatr Mex. 2017 mar;38(2):128-133.

49. Torres K, Sevilla E. Conceptos para la selección y uso de mascarillas y respiradores, como medidas de protección durante los brotes de influenza. Rev Inst Nal Enf Resp Mex. 2009;22(3):230-237.

50. Martins-Filho P, de Gois-Santos V, Tavares C, de Melo E, do Nascimento-Júnior E, Santos V. Recommendations for a safety dental care management during SARS-CoV-2 pandemic. Rev Panam Salud Publica. 2020;44:e51. DOI: 10.26633/RPSP.2020.51.

51. Liao L, Xiao W, Zhao M, Yu X, Wang H, Wang Q, et al. Can N95 Respirators Be Reused after Disinfection? How Many Times?. ACS Nano. 2020;14(5):6348-6356. DOI: $10.1021 /$ acsnano.0c03597. 
52. Cadnum J, Li D, Redmond S, John A, Pearlmutter B, Donskey C. Effectiveness of Ultraviolet-C Light and a High-Level Disinfection Cabinet for Decontamination of N95 Respirators. Pathog Immun. 2020;5(1):52-67. DOI: $10.20411 /$ pai.v5i1.372.

53. Araya S. Consideraciones para la atención de urgencia odontológica y medidas preventivas para COVID-19 (SARSCoV2). Int. J. Odontostomat. 2020;14(3):268270.

54. Ali S, Zeb U, Muhammad A. Transmission Routes and Infection Control of Novel Coronavirus-2019 in Dental Clinics - A Review. J Islamabad Med Dental Coll. 2020;9(1):63-70. DOI: 10.35787/jimdc.v9i1.517.

55. Van Doremalen N, Bushmaker T, Morris D, Holbrook M, Gamble A, Williamson B, et al. Aerosol and Surface Stability of SARS-CoV-2 as Compared with SARSCoV-1. N Engl J Med. 2020;382(16):1564-1567. DOI: 10.1056/NEJMc2004973.

56. Bustamante M, Herrera M, Ferreira. R, Riquelme D. Contaminación bacteriana generada por aerosoles en ambiente odontológico. Int. J. Odontostomat. 2014;8(1):99-105.

57. Badillo B, Morales G, Martínez C, Castillo U, Gasca N, Hernández G, et al. Análisis bacteriológico de piezas de mano de alta velocidad utilizadas en la práctica clínica. Rev ADM. 2019;76(5):261-266.
58. Li Y, Ren B, Peng X, Hu T, Li J, Gong T, et al. Saliva is a non-negligible factor in the spread of COVID-19 [published online ahead of print, 2020 May 4]. Mol Oral Microbiol. 2020;10.1111/omi.12289. DOI: 10.1111/ omi.12289.

59. Kampf G, Todt D, Pfaender S, Steinmann E. Persistence of coronaviruses on inanimate surfaces and their inactivation with biocidal agents. J Hosp Infect. 2020;104(3):246-251. DOI: $10.1016 /$ j. jhin.2020.01.022.

60. Bedell K, Buchaklian AH, Perlman S. Efficacy of an Automated Multiple Emitter Whole-Room Ultraviolet-C Disinfection System Against Coronaviruses MHV and MERS-CoV. Infect Control Hosp Epidemiol. 2016;37(5):598-599. DOI: 10.1017/ice.2015.348

61. Kariwa H, Fujii N, Takashima I. Inactivation of SARS coronavirus by means of povidone-iodine, physical conditions and chemical reagents. Dermatology. 2006;212 Suppl 1(Suppl 1):119-123. DOI: 10.1159/000089211.

62. Darnell ME, Subbarao K, Feinstone SM, Taylor DR. Inactivation of the coronavirus that induces severe acute respiratory syndrome, SARS-CoV. J Virol Methods. 2004;121(1):85-91. DOI: $10.1016 /$ j.jviromet.2004.06.006. 